\title{
A complete cell search and synchronization in LTE
}

\author{
Sriharsha M. R.* (D), Sreekanth Dama and Kiran Kuchi
}

\begin{abstract}
The initial process of identifying any available base station (BS) by a user equipment (UE) that wants to communicate is termed as cell search. To ensure a reliable communication, any UE has to be synchronized with the BS both in time and frequency domains. Cell search process is said to be complete once cell ID associated with long-term evolution (LTE) BS is decoded successfully. This paper presents a series of cell search and synchronization algorithms, which efficiently estimated time and frequency offsets as well as cell ID. The synchronization signals present in LTE, namely, primary synchronization signal (PSS) and secondary synchronization signal, that carry cell ID are critically exploited in the algorithms. The aforementioned algorithms are classified into two modules, namely, module I and module II, based on their computation complexity.

In module I, a cyclic prefix (CP)-based maximum likelihood (ML) estimator is employed to obtain a coarse estimate of time and fractional frequency offset; however, the estimates are refined using synchronization signals. A joint estimation of timing, integer frequency offset (IFO), and PSS ID (sector ID) is carried out in module II. Both the modules operate on the cross-correlation approach of PSS with the received signal for obtaining timing and sector ID. IFO as a part of module II is detected from a finite hypotheses set using synchronization signals. Extensive simulations are carried out on a time-varying frequency-selective channel to analyze the performance of the algorithms.
\end{abstract}

Keywords: LTE, Synchronization, Cell search, CFO

\section{Introduction}

Third generation partnership project (3GPP) has developed long-term evolution (LTE) technology to achieve high data throughput and better spectrum utilization. Supporting varied range of bandwidths from 1.4 to $20 \mathrm{MHz}$ makes this technology more flexible. LTE aims at data rates up to $100 \mathrm{Mbps}$ in down-link (DL) and $50 \mathrm{Mbps}$ in up-link (UL) with a bandwidth (BW) of $20 \mathrm{MHz}$ excluding carrier aggregation and spatial multiplexing [1]. Orthogonal frequency division multiplexing (OFDM) makes a perfect choice in the DL because of its competence in dealing channel frequency selectivity and its flexibility to handle different channel BWs. Single-carrier frequency division multiplexing (SC-FDM) is adopted in UL to reduce the peak to average power ratio (PAPR). A cyclic prefix (CP) is inserted in every OFDM and SC-FDM symbol. LTE base station (BS) supports normal and extended CP to combat delay spreads.

*Correspondence: ee13p0009@iith.ac.in

Electrical Engineering, ITH, Hyderabad, India
LTE cell operates on either time division duplexing (TDD) or frequency division duplexing (FDD) mode.

BS in a LTE cellular network are differentiated by their unique IDs. They are identified by 504 such distinct IDs called as cell ID. The deployment of these cells is done in such a way that the BSs having the same cell ID are placed far apart. The user equipment (UE) trying to communicate has to primarily decode the cell ID of the nearest BS. The process of attaining timing and frequency synchronization and cell ID of a BS is called as cell search [2]. Under this process of cell search, UE has to acquire basic information including cell ID, duplexing mode, timing, and frequency related to the BS.

\subsection{LTE frame and cell ID}

The data transmission in LTE is carried out with duration of $10 \mathrm{~ms}$ for each frame on a given bandwidth. Each frame is divided into 10 subframes of each $1 \mathrm{~ms}$ which are further divided into two slots of equal 
duration. Each slot consists of six or seven OFDM symbols depending on the $\mathrm{CP}$ length. A resource block (RB) is the smallest time-frequency resource unit which can be allocated to users. Each RB comprises of seven or six symbols with 12 resource elements in each symbol [3]. A resource element (RE) is the resource provided by one sub-carrier in an OFDM symbol.

The dedicated channel for synchronization in LTE is divided into two parts, namely, primary synchronization channel (PSCH) and secondary synchronization channel $(\mathrm{SSCH})$. The signals transmitted on PSCH are primary synchronization sequence (PSS) which carry sector ID (SID), and the signals on SSCH are secondary synchronization sequence (SSS) which carry group ID (GID). The cell ID is obtained by combining the IDs on PSS and SSS as given by

$$
\text { cellID }=3 * \text { GID }+ \text { SID } .
$$

In order to successfully detect the cell ID, UE has to extract SID and GID from both the synchronization channels. There are 504 distinct cell IDs available in LTE, which are grouped into 168 distinct groups identified by GID. All the groups contain three identical SIDs. GID $\in\{0,1,2 \ldots, 167\}$ is carried by SSS, and SID $\in\{0,1,2\}$ is carried by PSS.

Two OFDM symbols for each of PSCH and SSCH are transmitted in every frame with a separation of $5 \mathrm{~ms}$. PSS present in the two symbols convey the same message, i.e., SID. To differentiate the first and second half of a frame, SSS present in two symbols are not identical; however, they convey same information. The goal of the synchronization procedure is to align to the frame boundaries of corresponding BS. The cell search and synchronization process will be complete if the UE performs the following operations successfully.

1. Acquisition of the symbol and frame timing is the operation by which UE determines the start of each symbol, i.e., the precise set of samples that has to be fed to the DFT for OFDM demodulation. Frame timing determines the boundaries of the frame.

2. Carrier frequency offset (CFO) estimation involves synchronizing UE to BS carrier frequency by eliminating the frequency offsets generated at the RF section due to lossy oscillator or due to Doppler frequency shift.

3. The successful detection of cell ID by extracting the SID and GID along with duplexing mode and the CP length $\left(L_{\mathrm{CP}}\right)$.

After the completion of initial cell search, UE tries to decode broadcast data channel information which confirms the successful cell search procedure. Cell search procedure is said to be failed in case UE fails to decode the broadcast data channel.

\subsection{Synchronization signals}

The synchronization signals are placed in the center $1.08 \mathrm{MHz}$ of the bandwidth which corresponds to $72 \mathrm{sub}-$ carriers of the OFDM symbol. Primary synchronization sequence is constructed from one of the three length63 Zadoff-Chu (ZC) sequences in the frequency domain, with the center element punctured to avoid transmission on DC subcarrier [3]. Three PSS sequences are used in LTE, corresponding to the three SIDs. The sequence $S_{M}$ used for the PSS are generated from ZC sequence with selected roots $u=\{25,29,34\}$ and is given by

$$
S_{M}(k)=e^{\frac{-j u k(k+1)}{63}} ; \text { where } k=0,1, . ., 62 \text { and } M=0,1,2
$$

where $M$ is the SID. Similar to PSS, secondary sequence is also positioned in the centre 62 REs nullifying the DC subcarrier. The SSS is also a frequency domain sequence, which is an interleaved concatenation of two length-31 $\mathrm{m}$-sequences. The two sequences are named as even sequence and odd sequence. Both even and odd sequences are scrambled with an m-sequence whose cyclic shift value depends on sector ID. The odd sequence is further scrambled by an $\mathrm{m}$-sequence with cyclic shift value determined by even sequence. The combination of cyclic shifts of even sequence and odd sequence corresponds to the GID [3]. The synchronization signals are chosen based on their correlation properties and frequency offset sensitivity. The low sensitivity to frequency offsets by the signals helps in reducing the burden of synchronization on the devices. Without loss of generality, all the neighboring BSs are assumed to have distinct cell IDs. However, there are only three possible SIDs. So, there is a high probability that neighbouring BSs also possess same SID. The identification of cell-ID is confirmed by accurately decoding the broadcast channel. UE is expected to achieve a miss detection probability equal to $1 \%$ at $-6 \mathrm{~dB}$ SNR for broadcast channel [1].

\subsection{Motivation and related works}

The accurate synchronization in both time and frequency domains is gaining importance in the wake of new trends like carrier aggregation, HetNets, and coordinated multipoint [4]. Cell search and synchronization is a basic operation and a much power-consuming one in any receiver. UE has to sweep over wide range of bands to establish a connection with the BS. This makes the cell search process computationally complex. The computational complexity increases further with the incorporation of technologies like carrier aggregation in LTE. So, there is a need for efficient algorithms which can balance performance with the number of computations. 
There have been extensive studies on OFDM time and frequency synchronization. Works like $[5,6]$ have proposed estimators which make use of pilots broadcasted periodically. Two or more replicas of the $\mathrm{PN}$ sequences are used as the pilots in estimation of the CFO and timing. These pilots are transmitted within the coherence time. Assuming the channel is same between two pilots, the auto-correlation of the window with its replica will give the estimates of timing and frequency offsets. The pilot sequences used in LTE synchronization are ZC sequences whose structure is entirely different from the that of the sequences proposed in the prior works. The self-correlation method presented in [5] is not a practical choice in LTE because the transmission periodicity of the $\mathrm{ZC}$ signals is much higher than the coherence time. The coherence time of high-speed vehicles is much less than $5 \mathrm{~ms}$.

The work in [7] proposed ML estimator for timing and fractional portion of CFO (FFO) of an OFDM signal. This is accomplished by the autocorrelation of $\mathrm{CP}$ present in the OFDM symbol. We make use of this estimator to find the coarse timing of the OFDM symbol and FFO for module-I algorithms. The autocorrelation among the received samples will result in more noise terms yielding poor performance at low SNR. Averaging over multiple OFDM symbols will give better estimates of timing and FFO. The performance of this estimator is limited by the delay spread of the channel.

The works $[8,9]$ present algorithms for PSS timing and SID detection. These algorithms are based on crosscorrelation with all the possible PSS sequences generated at UE. Few of the above proposed algorithms follow the approach to estimate SID through time domain operations and few are based on frequency domain operations. The cross correlation approach in time domain would result in the sum of the exponentials in the presence of large CFO. It would affect the timing estimator unless the problem of large frequency offsets is addressed. The frequency domain estimation of SID presented in [10] makes the process computationally exhaustive by using DFT. Timing errors present if any would also have significant effect on the estimates in the frequency domain. Choosing the precise set of window of samples that has to be fed to DFT would be ambiguous because few of the OFDM symbols have $L_{\mathrm{CP}}$ of 160 instead of 144 . The DC algorithm presented in [11] estimated timing using autocorrelation of PSS present twice in a frame and tried to exploit the diversity by the neighboring sectors. The diversity is exploited from the multiple neighbor sectors having the same SID. The SID is detected by differential correlation of the frequency domain data with the possible PSS sequences. The exploitation of diversity could only be possible if all the sectors of different BSs are strongly synchronized in time and frequency. The channel effects may not be the same on both PSS which are half a frame apart under high Doppler conditions. Similarly, CCSA method proposed in [9] uses the CP-based method for timing and FFO detection. Using the obtained estimates, SID is detected using the frequency domain correlation with PSS sequences. The timing and frequency selectivity effects propagates through the algorithm leading to the performance loss.

Most of the previous works like [10] and [11] and others have not generalized the case for different $\mathrm{CP}$ lengths and TDD/FDD modes. The algorithms including [9] and [12] were also built on the assumption of known $L_{\mathrm{CP}}$.

The algorithms presented in this work are divided into two modules as module I and module II based on their computational complexity. In this paper, we propose a sequential execution of module I and module II to reduce the computational burden at the receiver. The algorithms allow to relax the oscillator restrictions as it involves the search of CFO over a large interval. During initial synchronization, devices have to sweep over a huge number of frequency bands. The devices like relays in which the oscillators are more precise do not undergo CFO effects. In the case of normal UEs, there is high probability of having hardware impairments and being less tolerant to temperature and ageing effects. The Doppler effect which contributes to the CFO will also be significant in the case of devices moving with high speeds.

CP-based approach is used to estimate coarse timing and FFO in module I, and the estimates are refined in time domain using PSS. However, if integer CFO is present in the received signal, it will affect the estimates of module I. So, module II which is a joint timing, integer CFO, and SID estimator is used in nullifying the CFO effect. Module II is resilient to the effects caused by integer $\mathrm{CFO}$ and large delay spreads since it is based on the cross-correlation with the PSS sequence over multiple integer CFO hypotheses. The residual offsets of CFO and timing are compensated using SSS and cell-specific reference signals (pilots). The proposed algorithms are simulated and evaluated under different delay spread conditions.

The remainder of this paper is as follows. The system model and problem statement are presented in Section 2. Timing, CP length, and SID and GID estimations are elaborated in Section 3 which also describes the module I and module II algorithms. Simulations conducted to study the performance of presented algorithms are explained in Section 4. Concluding remarks are presented in Section 5.

Notations: The notation $\|$.$\| indicates the norm of the$ enclosed vector. The signals in capital letters denote frequency domain. The notation $\Re \in$ \{\} represents the real part of the complex quantity. Matrices and vectors are written in bold letters. Notation |.| is used to show the cardinality of a set. 


\section{System model}

The transmitted signal from the target BS $x(n)$ is an OFDM-modulated signal obtained by assigning the symbols $X(k)$ to the subcarrier $k$. Each $X(k)$ is acquired by mapping the bits to symbols. $N$ point IDFT of the $X(k)$ symbols will yield the OFDM-modulated signal where $N$ depends on the system bandwidth. The continuous time OFDM-modulated signal given in (1) is transmitted over the carrier frequency $\left(f_{c}\right)$.

$$
x(t)=\sum_{k=-N / 2}^{-1} X(k) e^{\frac{j 2 \pi k\left(t-L_{C P} T_{s}\right)}{N T_{S}}}+\sum_{k=1}^{N / 2} X(k) e^{\frac{j 2 \pi k\left(t-L_{C P} T_{S}\right)}{N T_{S}}}
$$

The received signal at the UE is down-converted and filtered to obtain the baseband signal. The time domain baseband continuous signal $y(t)$ received at UE includes the effects of channel, CFO $(\Delta f)$, and thermal noise $(w(t))$ as given in (2). The signal is sampled at standard sampling rate of 30.72 Msps to obtain $y(n)$.

$$
y(t)=(x(t) * h(t)) e^{j 2 \pi \Delta f t}+w(t) .
$$

where $h(t)$ is the channel.

$$
y(n)=(x(n) \otimes h(n)) e^{\frac{j 2 \pi \epsilon n}{N}}+w(n)
$$

$h(n)$ is the equivalent discrete baseband channel. The CFO is normalized with the sub-carrier spacing and is given by

$$
\epsilon=\frac{\Delta f}{1 /\left(N T_{s}\right)} \text {. }
$$

The causes of CFO are due to mismatch between oscillators at BS and UE as well as due to Doppler shift. The integer (multiple of subcarrier spacing) and fractional (fractional portion of subcarrier spacing) parts of $\epsilon$ are separately estimated for computational convenience. The integer CFO (IFO) induces subcarrier shift, whereas the fractional CFO (FFO) leads to inter-carrier interference (ICI) and common phase error. The frequency offset present at any BS is very small compared to that of an UE, so the CFO contribution from the BS is not considered.

In this context, our aim is to extract the parameters SID, GID, and precise timing from the received signal given in (3). This process also includes the estimation and compensation of $\epsilon$ by locating the PSS and SSS symbol in the frame. However, the presence of multi-path channel $h(n)$ and the additive noise pose a challenge. The whole set of operations applied on the received signal to detect the required parameters is presented in Fig. 1. The operations are as follows:

1. The proposed algorithm performs the CP-based correlation according to [7] to obtain the coarse timing of an OFDM symbol and FFO.

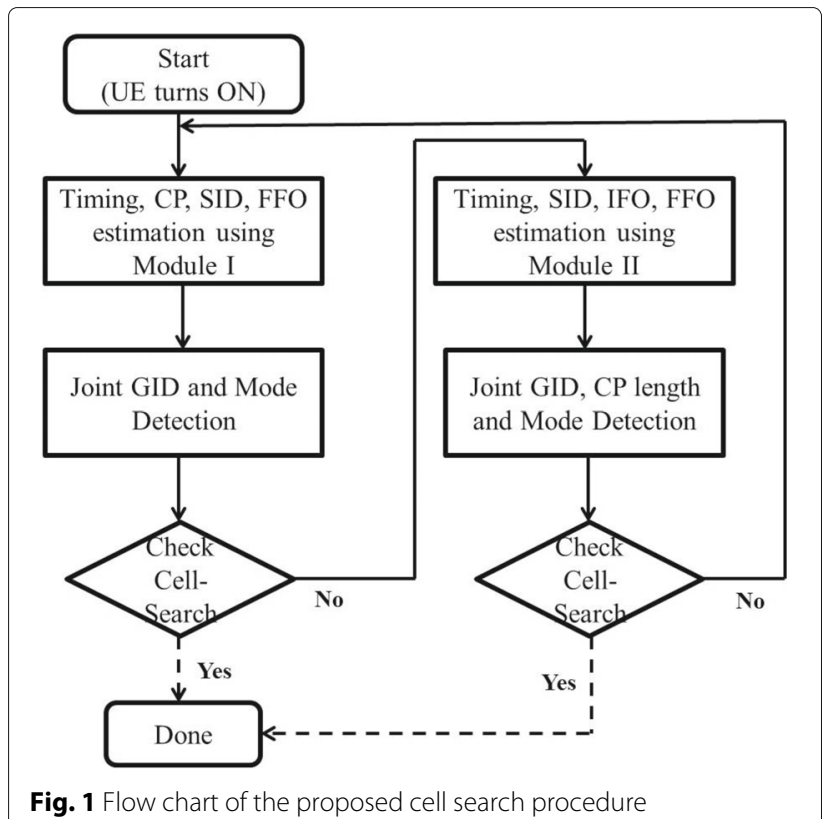

Fig. 1 Flow chart of the proposed cell search procedure

2. Based on the coarse results, fine timing, SID, and $L_{\mathrm{CP}}$ are obtained by the cross-correlation of the PSS over decimated received signal, further refined over standard sampling rate.

3. After sector ID detection, SSS ID and mode are detected based on the non-coherent receiver. Once SSS ID is detected, UE will be adjusted to the frame timings.

The above steps form the module I of the receiver section. If the receiver fails to complete the cell search, then UE performs the module II set of operations. They start with

4. Joint timing, SID along with IFO are detected based on ML-like search similar to that on [13].

5. FFO is estimated using the detected PSS by finite hypotheses search approach.

6. Finally, GID of the SSS along with $L_{\mathrm{CP}}$ are detected to complete the cell search process.

\section{Timing and SID detection}

The timing and SID detection of the received signal based on the two modules are explained in this section. Since the synchronization signal occupies $1.08 \mathrm{MHz}$ BW at the center of the spectrum, the received signal is decimated and cell search operation is performed.

The output of the decimator $\hat{y}(n)$ is given by

$$
\tilde{y}(n)=y(V n)
$$

where $\tilde{y}(n)$ is filtered and decimated output of $y(n), V$ is the decimating factor. 


\subsection{Module I}

Module I is the set of computationally efficient operations for the estimation of CFO, timing, SID, and $L_{\mathrm{CP}}$. This set of algorithms assumes the CFO present is only a fractional offset. CP-based estimator for CFO is used, and the estimator is averaged over multiple symbols. SID and timing are obtained by cross-correlating with the possible PSS sequences.

\subsubsection{Coarse timing and FFO estimation}

The maximum likelihood (ML) estimator for timing and FFO estimation of an OFDM-based system is proposed in [7]. The method makes use of the redundancy present in each symbol in terms of $\mathrm{CP}$ to estimate the timing as well as FFO. The channel is assumed to be constant within one OFDM symbol which is a valid assumption. The coarse timing estimate $\left(\hat{\tau}_{c}\right)$ and FFO estimate $\left(\hat{\epsilon}_{f}\right)$ from the received non-decimated signal are estimated using this method. The estimate could be averaged over multiple symbols for better results. The implementation of this method starts with auto-correlation of a window of CP length samples with signal of $N$ samples apart and moving the window sample by sample. The coarse timing and FFO is obtained from the log likelihood function according to the below set of equations.

$$
\begin{aligned}
& \hat{\tau}_{c}=\underset{n}{\operatorname{argmax}}\{2 \gamma(n)-\rho \varepsilon(n)\} \\
& \hat{\epsilon}_{f}=\frac{-1}{2 \pi} \angle \gamma\left(\hat{\tau}_{c}\right)
\end{aligned}
$$

where $\gamma(n)$ is the correlation sum and

$$
\gamma(n)=\sum_{k=n}^{n+L_{C P}-1} y(k) y^{*}(k+N)
$$

$\varepsilon(n)$ is the energy term

$$
\varepsilon(n)=\sum_{k=n}^{n+L-1}\|y(k)\|^{2}+\|y(k+N)\|^{2}
$$

$\|$.$\| represents the norm of the complex sample. \rho$ is a weighing factor obtained by the ratio of signal energy $\left(\sigma_{s}^{2}\right)$ to signal plus noise energy $\left(\sigma_{n}^{2}\right)$.

$$
\rho=\frac{\sigma_{s}^{2}}{\sigma_{s}^{2}+\sigma_{n}^{2}}
$$

\subsubsection{Timing alignment, $S I D$, and $L_{C P}$ detection}

The estimates $\left(\hat{\tau}_{c}, \hat{\epsilon}_{f}\right)$ obtained from Section 3.1.1 are used in refining the LTE frame boundary estimates. As the two PSS sequences in a frame are same, the location estimated using PSS gives the boundaries of half-a-frame. Before performing any fine estimation on PSS timing $(\hat{\tau})$, the
FFO present in the received signal is corrected using the estimates obtained from (4).

$$
\tilde{y}_{f}(n)=\tilde{y}(n) e^{\frac{-j 2 \pi \hat{\epsilon}_{f} n}{N}}
$$

An observation window of half-a-frame duration is considered for PSS, $L_{\mathrm{CP}}$, and timing detection. The detection of $L_{\mathrm{CP}}$ is done by arranging the samples into two different formats of the CP length. $N_{H F}^{D}$ are the number of samples in half-a-frame obtained after decimating the received signal. Two sets are constructed using these samples. One is a set of 70 OFDM symbols each with normal CP and the other is a set of 60 OFDM symbols each with extended CP. $\Upsilon_{1}$ and $\Upsilon_{2}$ are the sets each containing 70 and 60 OFDM symbols corresponding to normal and extended CPs respectively.

The operations carried out in this section are shown in Fig. 2.

The elements of $\Upsilon_{1}$ are

$$
\left\{\Upsilon_{1}\right\}=\left\{\mathbf{X}_{0}, \mathbf{X}_{1}, \ldots \mathbf{X}_{69}\right\}
$$

where each $\mathbf{X}_{k}$ is an OFDM symbol with normal CP. $p_{k}(n)$ are the samples collected from $\tilde{y}_{f}(n)$, and are arranged as OFDM symbols to form $\mathbf{X}_{k}$.

$\mathbf{X}_{k}=\left[p_{k}(0), p_{k}(1), \ldots, p_{k}\left(N^{D}+L_{C P 1}^{D}-1\right)\right]$; for $\mathrm{k}=0,1, . .69$ where $N^{D}$ and $L_{C P 1}^{D}$ are symbol and normal CP sizes of an OFDM symbol after decimation. For the PSS timing and ID, each $\mathbf{X}_{k}$ after eliminating CP is cross-correlated with the time domain PSS signals generated at the receiver. The procedure is repeated for all the possible values of $M$. The time domain PSS signal is generated as in (6)

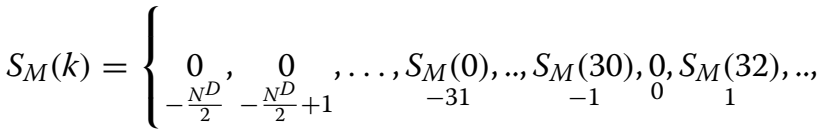

$$
\begin{aligned}
& \left.\begin{array}{lcc}
S_{M}(62), . ., & 0 & 0 \\
31 & \frac{N^{D}}{2}-2 & \frac{N^{D}}{2}-1
\end{array}\right\} \\
& s_{M}(n)=\sum_{k=-\frac{N^{D}}{2}}^{-1} S_{M}(k) e^{\frac{j 2 \pi k n}{N^{D}}}+\sum_{k=1}^{N^{D} / 2} S_{M}(k) e^{\frac{j 2 \pi k n}{N^{D}}}
\end{aligned}
$$

The correlation sums $\left(\Xi_{M}^{1}\right)$ are obtained for all the OFDM symbols in $\Upsilon_{1}$ and is given by

$$
\Xi_{\mathbf{M}}^{1}(q)=\frac{1}{N^{D}} \sum_{n=L_{C P 1}^{D}}^{N^{D}+L_{C P 1}^{D}-1} p_{q}(n) s_{\mathbf{M}}^{*}(n)
$$

the $p_{q}(n)$ is the OFDM symbol after compensating estimated frequency offsets

$$
p_{q}(n)=\sum_{l=0}^{l=\vartheta-1} h(n-l) x(l)+\tilde{w}(n) .
$$

$\vartheta$ is the number of channel taps. 


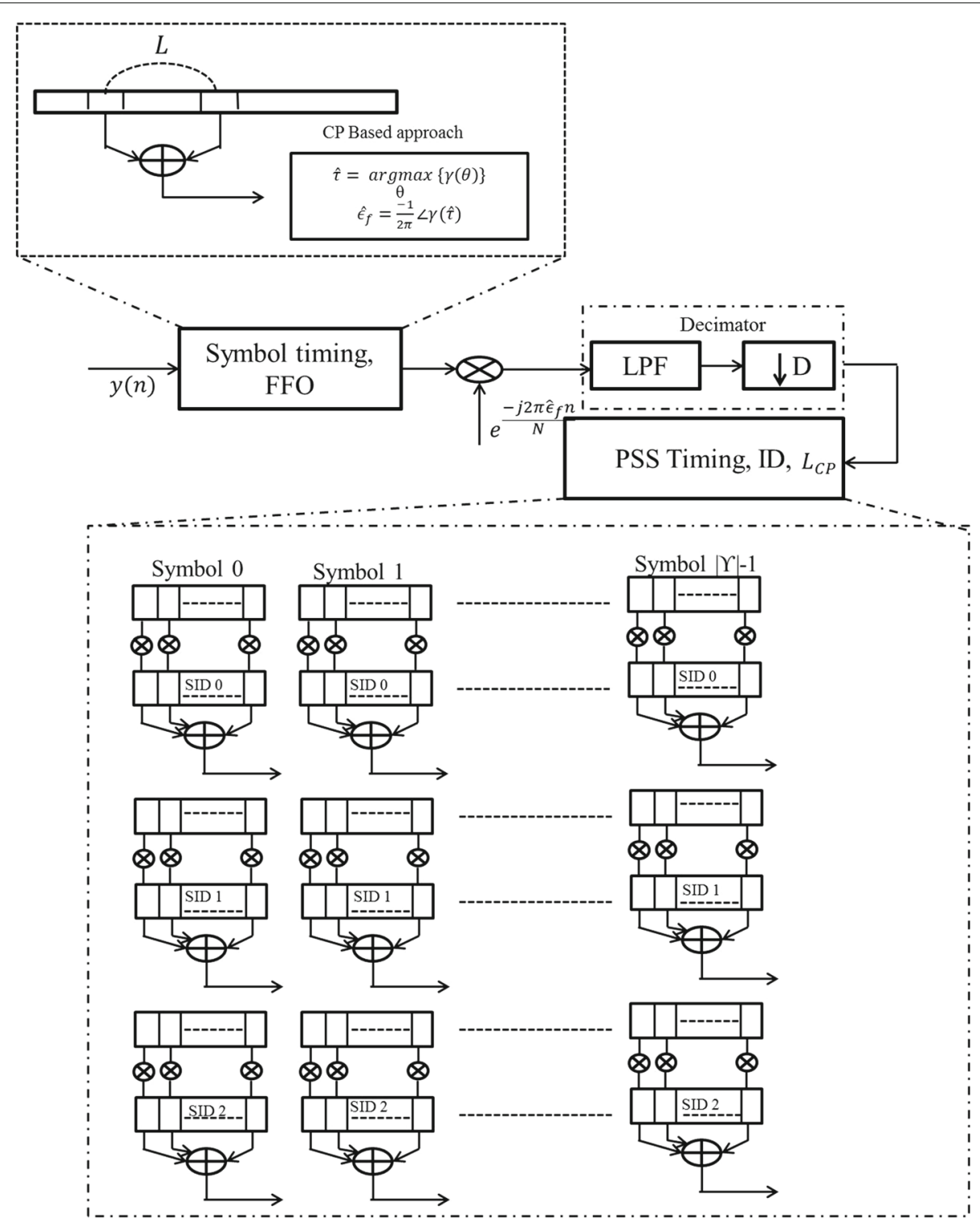

Fig. 2 Complete procedure of module I explained graphically

Similarly, the set $\Upsilon_{2}$ is prepared with the $N_{H F}^{D}$ samples according to extended CP $\left(\left|\Upsilon_{2}\right|=60\right)$. $\left\{\Upsilon_{2}\right\}=\left\{\mathbf{X}_{0}, \mathbf{X}_{2}, \ldots \mathbf{X}_{59}\right\}$; each $\mathbf{X}_{k}$ is an OFDM symbol with extended $C P\left(L_{C P 2}^{D}\right)$. The set of operations carried on $\Upsilon_{1}$ with $s_{M}(n)$ has to be repeated for $\Upsilon_{2}$.

Similarly, the set $\Upsilon_{2}$ prepared with the same $N_{H F}^{D}$ samples according to extended $\mathrm{CP}$ is used in calculating the correlation energy $\Xi_{\mathbf{M}}^{2}$.

$$
\Xi_{\mathbf{M}}^{2}(q)=\frac{1}{N^{D}} \sum_{n=L_{C P 2}^{D}}^{N^{D}+L_{C P 2}^{D}-1} p_{q}(n) s_{\mathbf{M}}^{*}(n)
$$

The obtained energy terms from two sets are used in identifying the SID, PSS timing, and $L_{\mathrm{CP}}$. From (7), (8), and (9), $\Xi_{\boldsymbol{M}}$ for a given SID and fixed CP index can be written as

$$
\Xi_{\mathbf{M}}(q)=\frac{1}{N^{D}} \sum_{n=0}^{N^{D}-1}\left\{\sum_{l=0}^{l=\vartheta-1} h(n-l) p_{q}(l)+w(n)\right\} s_{\mathbf{M}}^{*}(n)
$$

Consider $p_{q}(n)$ as the samples constituting PSS symbol of the considered SID. Then, $\Xi_{\mathbf{M}}(q)$ can be written as

$$
\Xi_{\mathbf{M}}(q)=\frac{1}{N^{D}} \sum_{n=0}^{N^{D}-1}\left\{\sum_{l=0}^{l=\vartheta-1} h(n-l) s_{M}(l)+w(n)\right\} s_{\mathbf{M}}^{*}(n)
$$


The frequency domain representation of $\Xi_{\mathbf{M}}(q)$ is the convolution of the received signal with the $S_{M}^{*}(-k)$ as given in (12).

$$
\Xi_{\mathbf{M}}=\frac{1}{N^{D}}\left\{H(k) S_{M}(k)+W(k)\right\} \otimes S_{M}^{*}(-k)
$$

Since we know from the properties of ZC sequences (13), $\Xi_{\boldsymbol{M}}$ is maximum for the PSS symbol.

$$
\frac{1}{N} \sum S_{M}(k) S_{M}^{*}(k+j)=\left\{\begin{array}{l}
0 ; \text { if } j \neq 0 \\
1 ; \text { if } j=0
\end{array}\right.
$$

The maximum energies estimated for both the $\mathrm{CP}$ are compared to detect the $L_{C P}$.

$$
\left\{\hat{M}, \hat{q}, \hat{L}_{C P}\right\}=\underset{M, q, i}{\operatorname{argmax}}\left\{\left\|\Xi_{\mathbf{M}}^{i}(q)\right\|^{2}\right\}
$$

The maximization of $\Xi$ over $q, M$, and $i$ will give the PSS the timing location, SID, and $L_{\mathrm{CP}}$ as in (14). Where $i$ is the index running over the CP size prospects. The timing obtained in (14) is based on the decimated sample of $y(n)$. The timing is refined over the estimate obtained using $\hat{M}$ and the PSS location. For fine timing estimate, cross-correlation is applied over $y(n)$ with the PSS of $\hat{M}$ on standard sampling rate.

\subsection{Module II}

The estimation of IFO is not considered in module I approach. The presence of IFO will certainly affect the PSS timing estimation. The correlation energy $(\Xi)$ is maximized in Section 3.1.1. The samples of $q^{\text {th }}$ OFDM symbol $p_{q}$ used in estimation of $\left(\Xi_{\mathbf{M}}(q)\right)$ are free from FFO. But the uncompensated IFO present will result as $\Xi_{\mathbf{M}}(q)$ as in (15).

$\Xi_{\mathbf{M}}(q)=\frac{1}{N^{D}} \sum_{n=0}^{N^{D}-1}\left\{\sum_{l=0}^{l=\vartheta} h(n-l) p_{q}(l) e^{\frac{j 2 \pi \epsilon_{i} n}{N^{D}}}+w(n)\right\} s_{\mathbf{M}}^{*}(n)$

Where $\epsilon_{i}$ is the IFO present in the collected samples. Unless the IFO is compensated from the received signal, maximization of the metric $\boldsymbol{\Xi}_{\mathbf{M}}$ does not yield the expected results. The coarse timing and FFO are estimated using CP in Section 3.1.1 and refined using the PSS sequence. The large delay spreads of the channel will affect the CP-based timing estimation, and the IFO presence influences the PSS timing estimation in module I. Thus, the error propagates through the PSS timing and SID estimation. So, to compensate the effects of IFO and delay spread, a joint PSS timing, SID, and IFO estimator is laid out as in [13]. In this module, the received signal $y(n)$ is decimated to $\tilde{y}(n)$ similar to module I. $N_{H F}^{D}$ samples of $\tilde{y}(n)$ are collected which contains at least one PSS sequence. The approach to estimate IFO follows by hypothesizing the IFO into finite number of possibilities $(\mu)$. From each SID, the $\mu$ number of PSS signals are generated by introducing one of the IFO hypotheses each time as in (16).

$$
s_{M}^{\delta}(n)=\sum_{k=-N_{D} / 2}^{-1} S_{M}(k-v) e^{\frac{j 2 \pi k n}{N_{D}}}+\sum_{k=1}^{N_{D} / 2} S_{M}(k-v) e^{\frac{j 2 \pi k n}{N_{D}}}
$$

where $v$ is the hypothesized IFO and $\delta \in\{0,1,2, . ., \mu-1\}$. ML approach for timing and SID in the presence of AWGN is obtained by maximization of the correlation of the received signal with the hypotheses. Thus, the correlation matrix is prepared with the collected $\tilde{y}(n)$ samples with all the $\delta$ hypotheses.

$$
R(l, \delta)=\sum_{n} \tilde{y}^{*}(n+l) s_{M}^{\delta}(n)
$$

The joint timing $(\hat{\tau})$, SID $(\hat{M})$, and IFO $(\hat{v})$ estimation is given by the maximization of the correlation sum obtained from (17).

$$
\{\hat{M}, \hat{\tau}, \hat{v}\}=\underset{M, l, \delta}{\operatorname{argmax}}\left\{\|R(l, \delta)\|^{2}\right\}
$$

The timing $(\hat{\tau})$ is refined using the non-decimated signal $y(n)$. A search window $(\alpha)$ is selected around $V \hat{\tau}$. The received signal $y(n)$ is correlated with the PSS of estimated SID on the standard sampling rate, and the refined timing is obtained by maximization of (19).

$$
\chi(l)=\sum_{n} y(V \hat{\tau}+l+n) s_{\hat{M}}(n)
$$

Where $l=\left\{\frac{-\alpha}{2}, \frac{-\alpha}{2}+1, \ldots, \frac{\alpha}{2}\right\} . L_{\mathrm{CP}}$ detection has to be done along with the SSS detection process in this set of algorithms. The computational complexity increases with the increase in $\mu$. Choosing $\mu$ as $5(v=\{-2,-1, . ., 2\})$ will result in 15 possible energy values of $R$. This approach is resilient towards the effects of large delay spread. Large delay spreads would affect the coarse timing in module I. 
Error propagates through fine PSS timing and SID from the affected coarse timing estimate. The PSS symbol is used in module II excluding the CP for the timing estimation. Similarly, the FFO estimation is also based on the PSS symbol without $C P$.

\subsubsection{FFO estimation}

In this section, the FFO estimation procedure is presented based on the correlation with PSS symbol. The timing and SID determined from Section 3.2 are used to estimate FFO. The PSS-based frequency offset estimation starts by compensating the received signal with the estimated IFO $\tilde{y}_{f}(n)=\tilde{y}(n) e^{\frac{-j 2 \pi \hat{v} n}{N_{D}}}$. Once the IFO is compensated, the uncertainty span of the CFO is $\epsilon_{f} \in[-0.5,0.5]$ which could be any real number. A near ML approach with a finite number of hypotheses is proposed in this section. $k_{f}$ finite number of frequency offset hypotheses that span the uncertainty of the CFO is considered for the FFO estimation. For each hypothesis test, corresponding amount of offset is removed from the $\tilde{y}_{f}(n)$ and correlated with the PSS signal of obtained SID. A hypothesis resulting the maximum correlation sum is the residual FFO.

The span of $\epsilon_{f}$ is first partitioned into number of hypotheses as $\left\{\epsilon_{f}^{0}, \epsilon_{f}^{1}, . ., \epsilon_{f}^{k_{f}-1}\right\}$. The uncertainty span of $\epsilon_{f}$ is hypothesized as $\left\{\epsilon_{f}^{0}, \epsilon_{f}^{1}, . ., \epsilon_{f}^{k_{f}-1}\right\}$. Now, each hypothesis is considered to obtain correlation energy as in (20), and maximum is identified. The flow of operations of FFO estimation is as follow

$$
\Lambda(i)=\sum_{n} \tilde{y}_{f}(n) e^{\frac{-j 2 \pi \epsilon_{f}^{i}}{N_{D}}} s_{\hat{M}}^{*}(n)
$$

- Prepare a set of finite number of hypotheses as $\left\{\epsilon_{f}^{0}, \epsilon_{f}^{1}, . ., \epsilon_{f}^{N_{f}^{D}-1}\right\}$ from the uncertainty span of FFO.

- Check the correlation sum by removing an amount equal to each of the offsets in the hypotheses from $\tilde{y}_{f}(n)$ as in (20). Obtain the offset $\left(\epsilon_{f}^{\hat{i}}\right)$ which maximizes the correlation sum.

$$
\hat{i}=\underset{i}{\operatorname{argmax}}\left\{\|\Lambda(i)\|^{2}\right\}
$$

- A new set of hypotheses is prepared based on the maximizing offset from the above step. Let us say $\epsilon_{f}^{2}$ maximizes the correlation energy, then the span of $\left[\epsilon_{f}^{1}, \epsilon_{f}^{3}\right]$ is further divided into $N_{f}^{D}$ equidistant hypotheses.

- The steps 2 and 3 are repeated to obtain the estimate of FFO $\left(\hat{\epsilon}_{f}\right)$. Simulations are stopped after four repetitions. The increase in the number of repetitions decreases the error variance of the estimate.

\section{Results and discussions}

Simulations were carried out to analyze the performance of the cell search and synchronization algorithms. The simulation conducted follows the LTE specifications of the signal generation and transmission. Since the synchronization signals are always transmitted on single antenna in LTE, only the SISO case is considered. During initial synchronization, UE starts the cell search process without any prior information related to LTE BS. Simulations are carried out on a frequency-selective fading channel in the presence of CFO, and performance at different SNRs (in decibels) is plotted.

International telecommunication union (ITU) has developed few channel models depending on the carrier frequency [14]. 3GPP further extended the ITU channel models to reflect the LTE channel characteristics at around $2.5 \mathrm{GHz}$ carrier frequency and $20 \mathrm{MHz}$ bandwidth. The Extended Pedestrian A(EPA), Extended Vehicular A (EVA), and extended typical urban (ETU) are the channel models based on the low, medium, and large delay spreads respectively [15]. The discrete time equivalent channel is obtained for the channel model based on the BW of $20 \mathrm{MHz}$. The discrete channel is similar to a tapped-delay-line filter with taps uncorrelated. Each tap of the channel is generated from Jakes spectrum [16]. The simulation setup is presented in Table 1.

\subsection{SID and timing detection}

The coarse timing in module I uses the CP-based estimator similar to [9]. The root mean square error (RMSE) of symbol timing is plotted in Fig. 3 which is obtained by averaging over seven OFDM symbols. The channel delay spread limits the performance of the CP-based timing estimator which can be observed as error floor in the plot. The performance degrades with increase in the delay spread of the channel.

The residual timing error contributes to the phase rotation in the frequency domain which can be compensated using the pilot symbols.

The detection probability for SID using CCSA is obtained, and the results are plotted in Fig. 4. The

Table 1 Simulation parameters

\begin{tabular}{ll}
\hline Parameter & Value \\
\hline Channel bandwidth & $20 \mathrm{MHz}$ \\
Channel model & EPA, EVA, ETU (ITU models) \\
Channel tap model & Jakes \\
No. of Tx antennas & 1 \\
No. of Rx antennas & 1 \\
Frame structure & Type II (TDD) \\
CFO introduced & $2.06(\times 15 \mathrm{KHz})$ \\
CP type & Normal
\end{tabular}




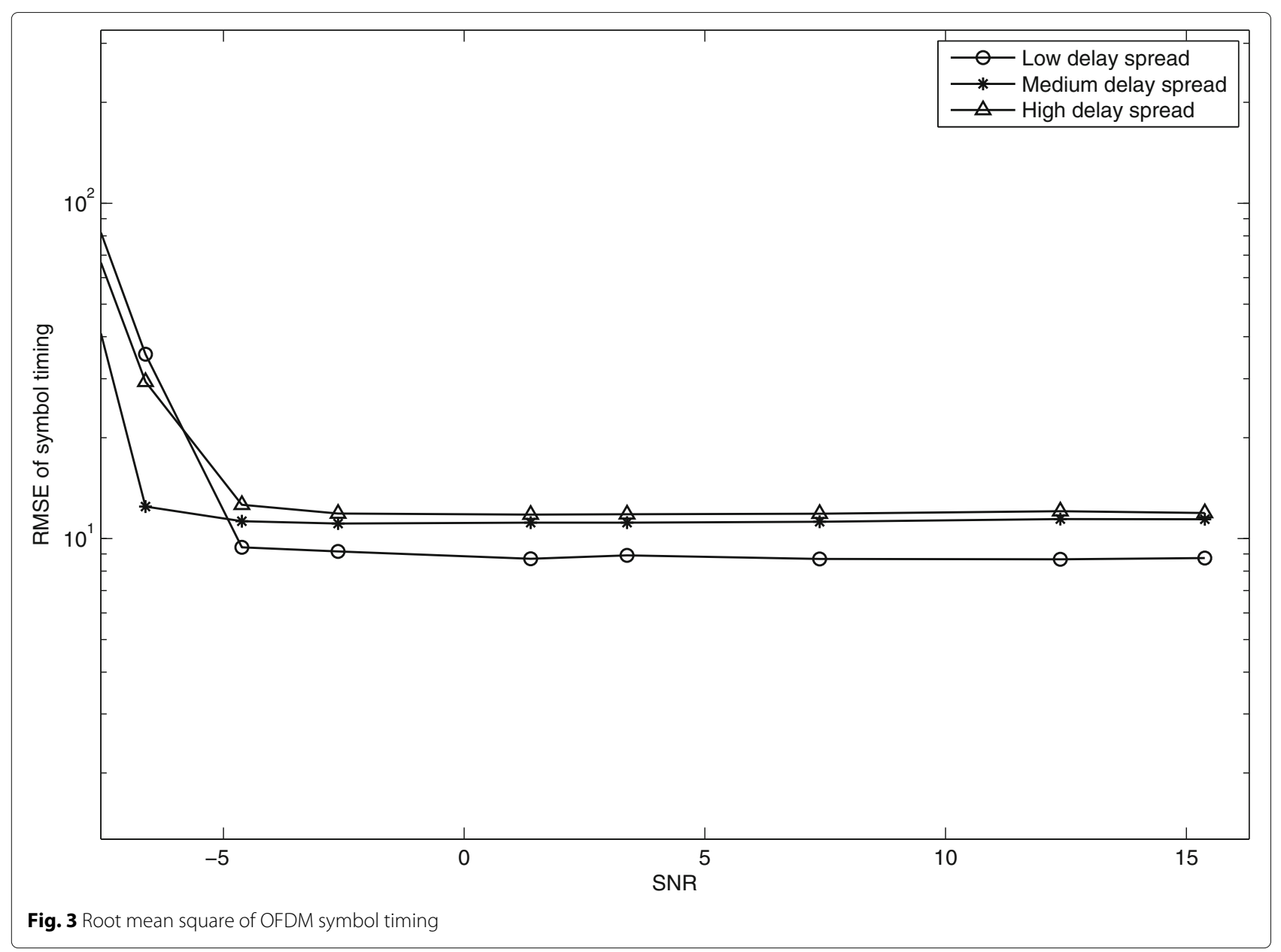

Fig. 3 Root mean square of OFDM symbol timing

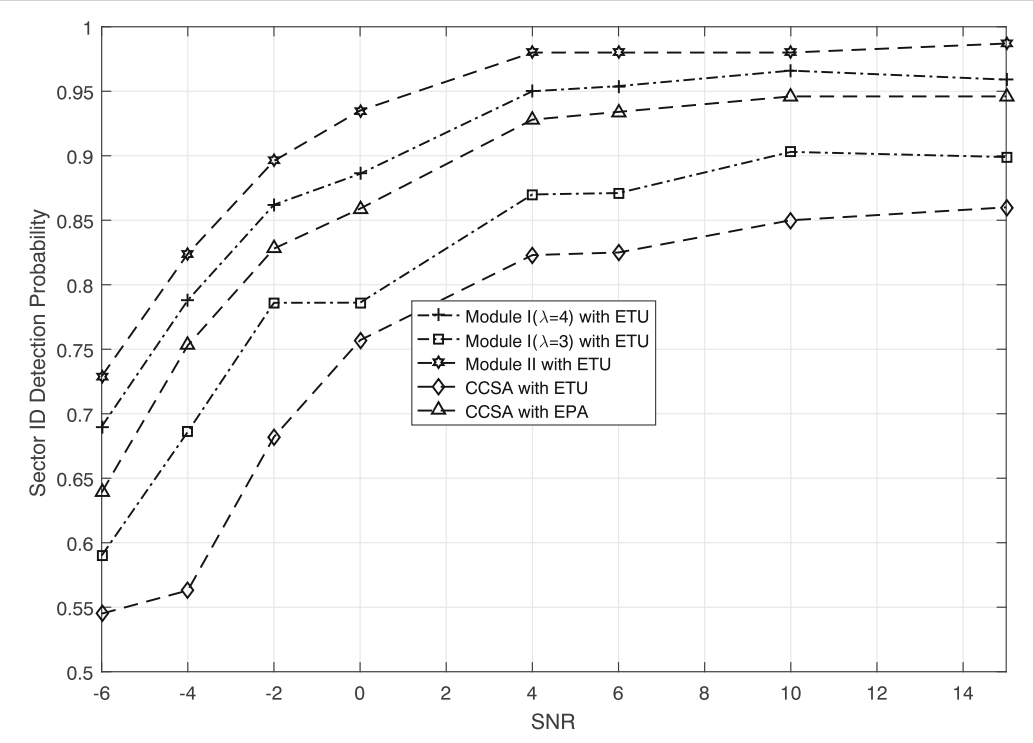

Fig. 4 SID detection probability using CCSA algorithm 


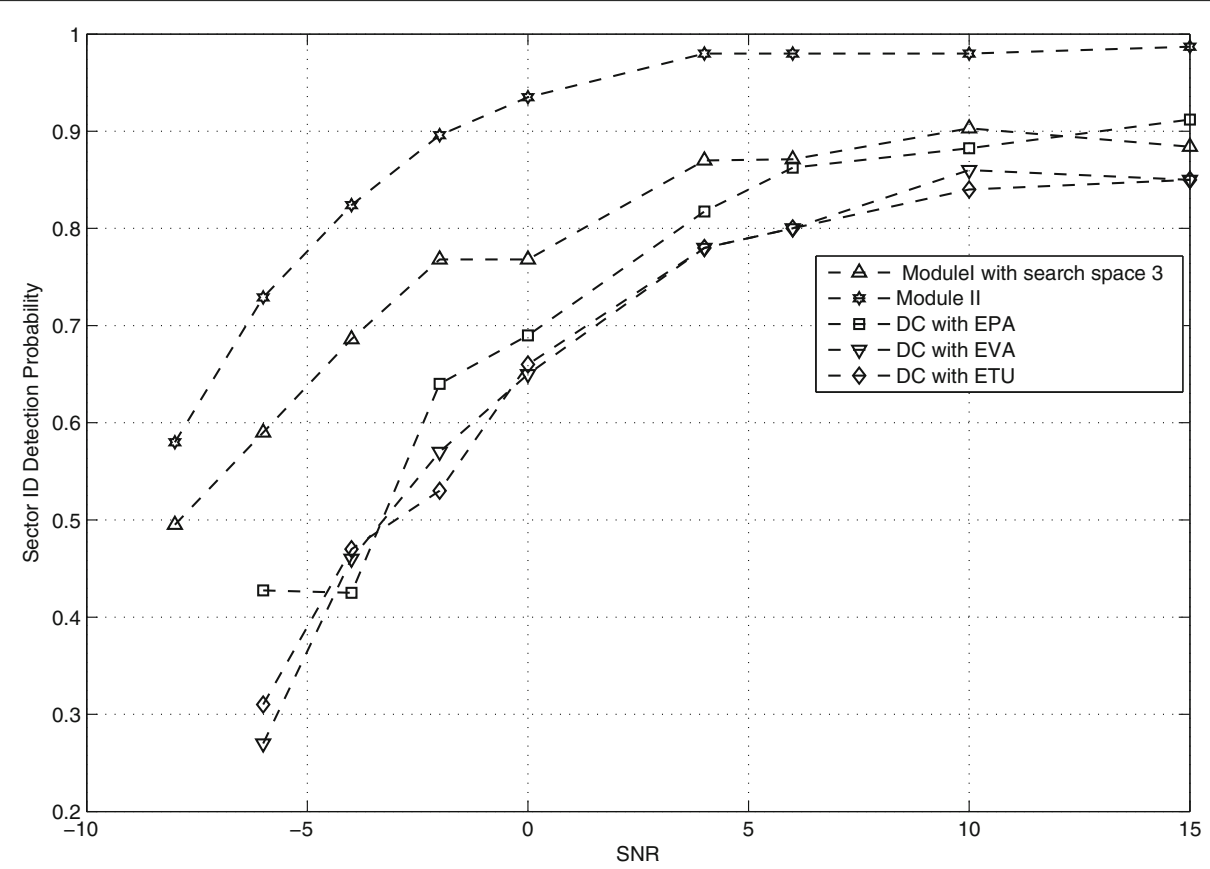

Fig. 5 SID detection probability using DC algorithm

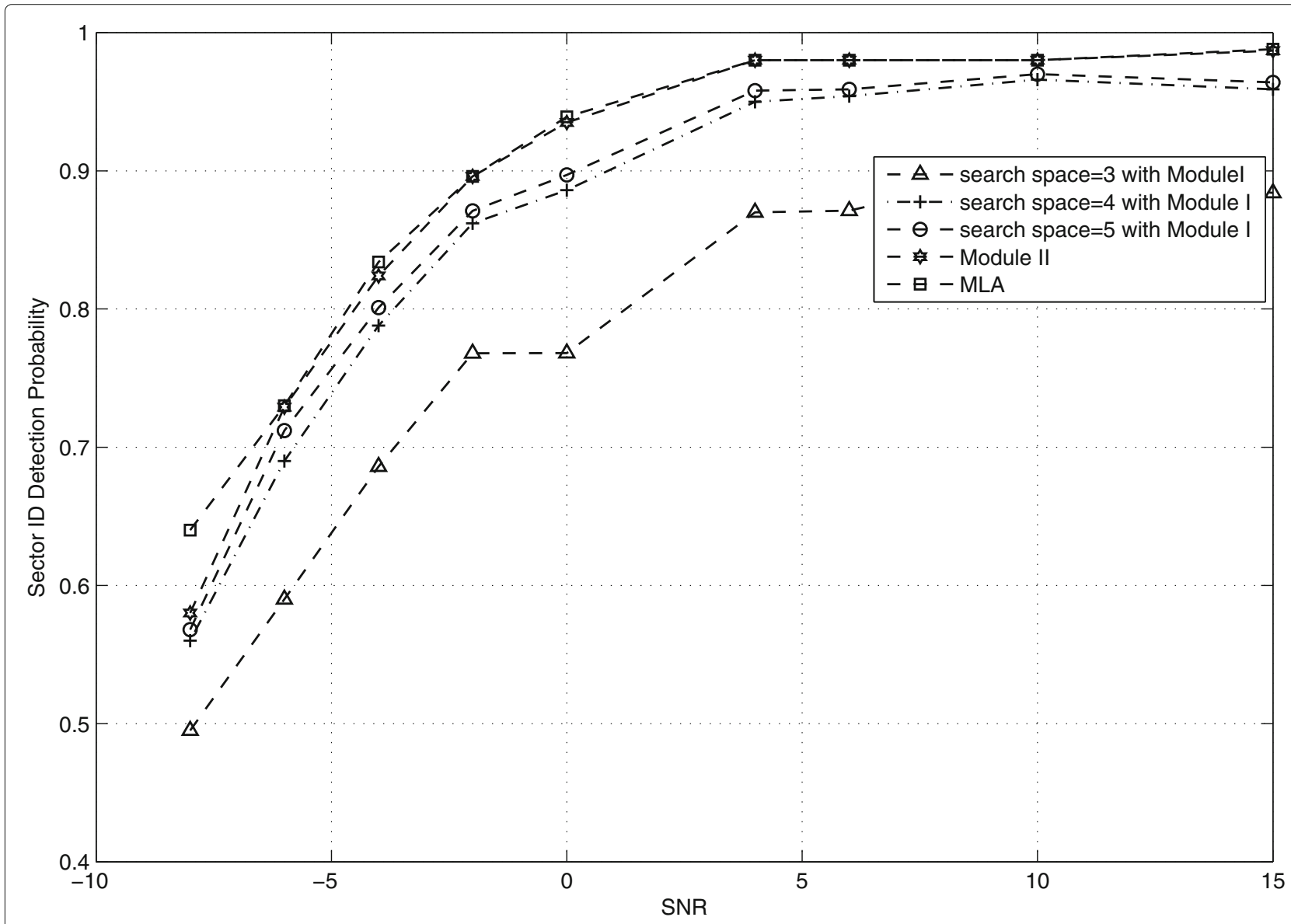

Fig. 6 Successful detection probability of SID 


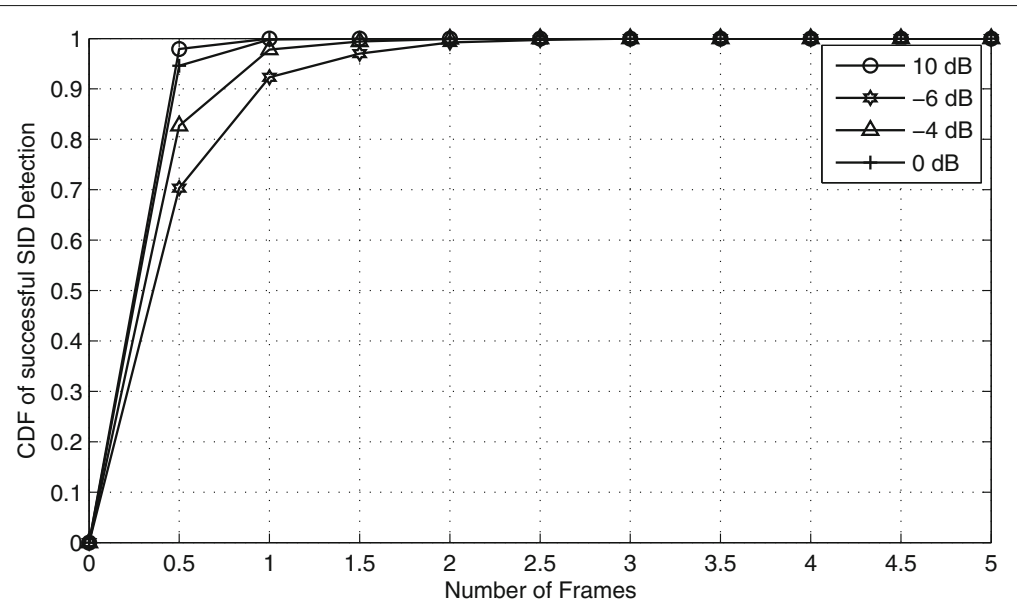

Fig. 7 CDF of the SID detection over number of frames

algorithm depends on the frequency domain correlation of the PSS sequences over the 62 subcarriers of each OFDM symbol. The algorithm is more prone to frequency selectivity effects as it depends on the direct correlation with the desired sequence in the frequency domain. It is evident from the figure that there is a performance loss of nearly $4 \mathrm{~dB}$ in the case of ETU channel with respect to the EPA channel. The results are compared with module I and module II. Module I with a search space $(\lambda)$ of 4 is considered for comparison in Fig. 4. Figure 5 shows the

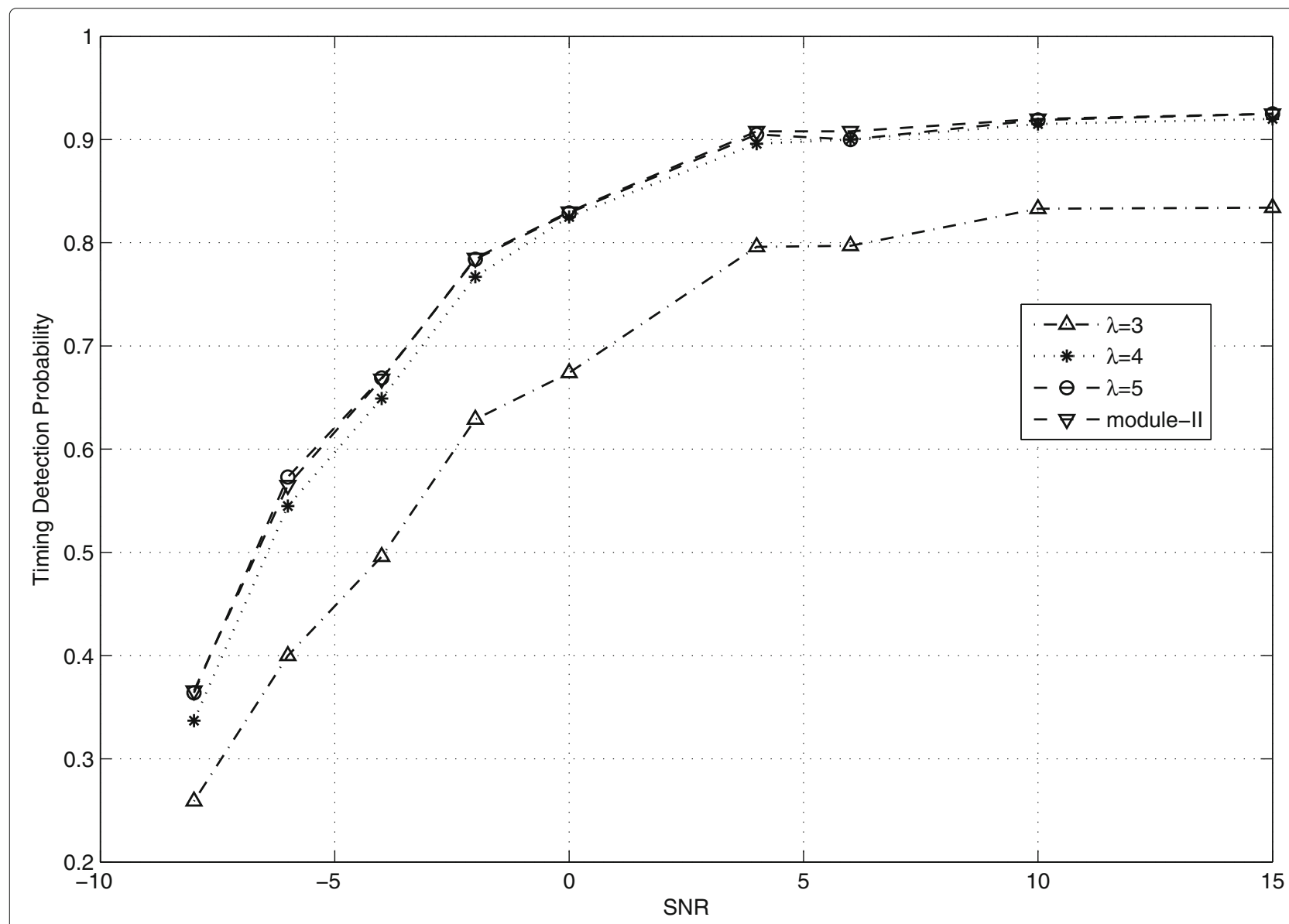

Fig. 8 Timing detection probability using module I and module II 


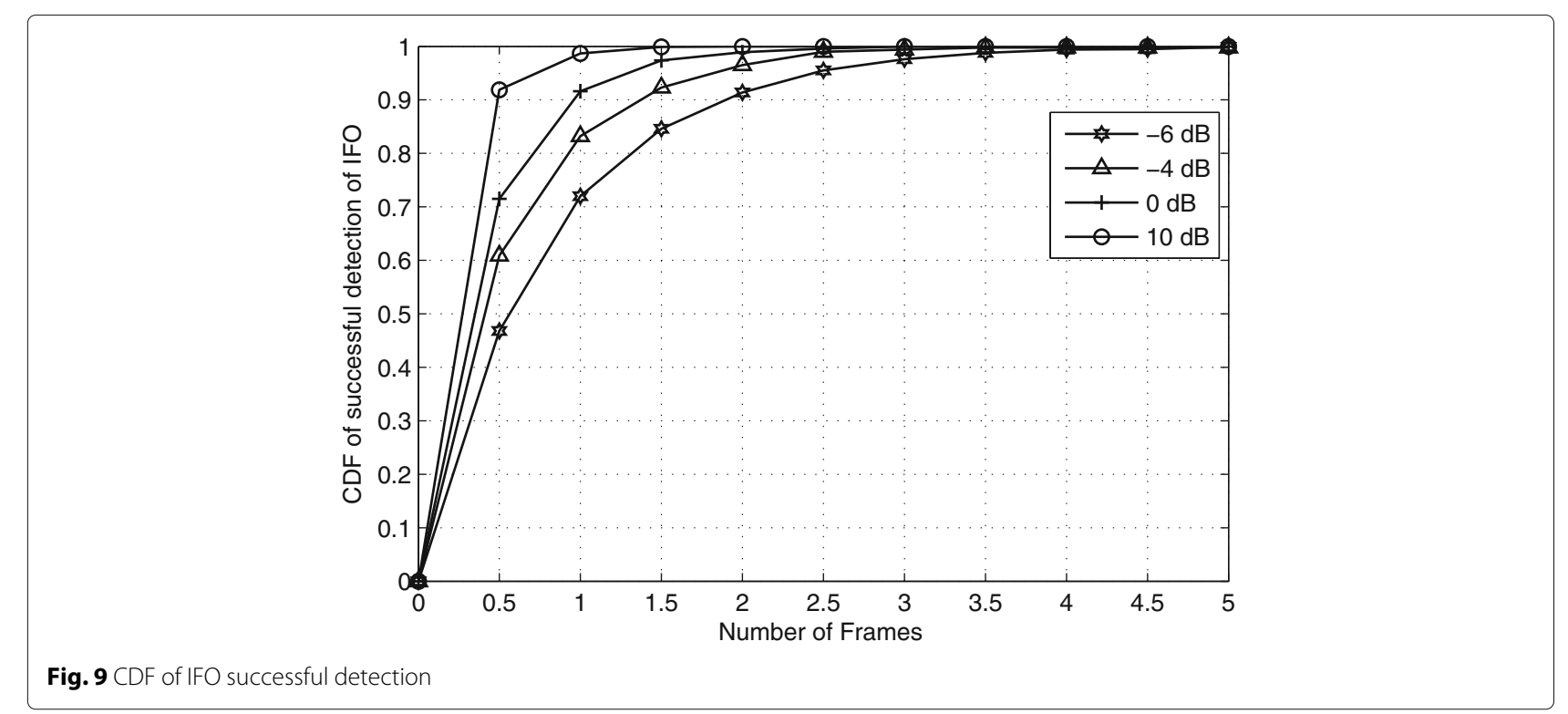

performance of the DC method on different channel conditions and compared with module I. The DC algorithm presented in [11] tries to estimate the timing using the two PSS sequences present in a frame. It follows the differential correlation of the received frequency domain signal with the PSS sequences for SID detection. The differential correlation among the consecutive subcarrier data will get rid of the frequency selectivity effects but allow performance loss at low SNR. The timing estimated using the PSS sequences separated by $5 \mathrm{~ms}$ limits the performance of this estimator.

The results of SID and timing detection based on both the modules are presented in Fig. 6. The cross-correlation of the received signal with the PSS sequences yields better results which can be observed here. Figure 6 shows the SID detection probability using module I and module II. In normal CP case, every first symbol of each slot has a CP of 160 samples instead of 144 . As module I is a symbolby-symbol search method, the extra 16 samples occurring has to be taken into account. The search window of each OFDM symbol is increased by $\lambda$ samples. $\lambda$ for the signal which is being operated on $V=16$ is one. The increase in the $\lambda$ will increase the window size yielding performance at the cost of computational complexity.

The simulations are carried out by varying $\lambda$ over 3,4 , and 5 and compared with module II and the MLA algorithm. MLA algorithm is the non-decimated version of module II. The results presented in Fig. 6 show that $\lambda$

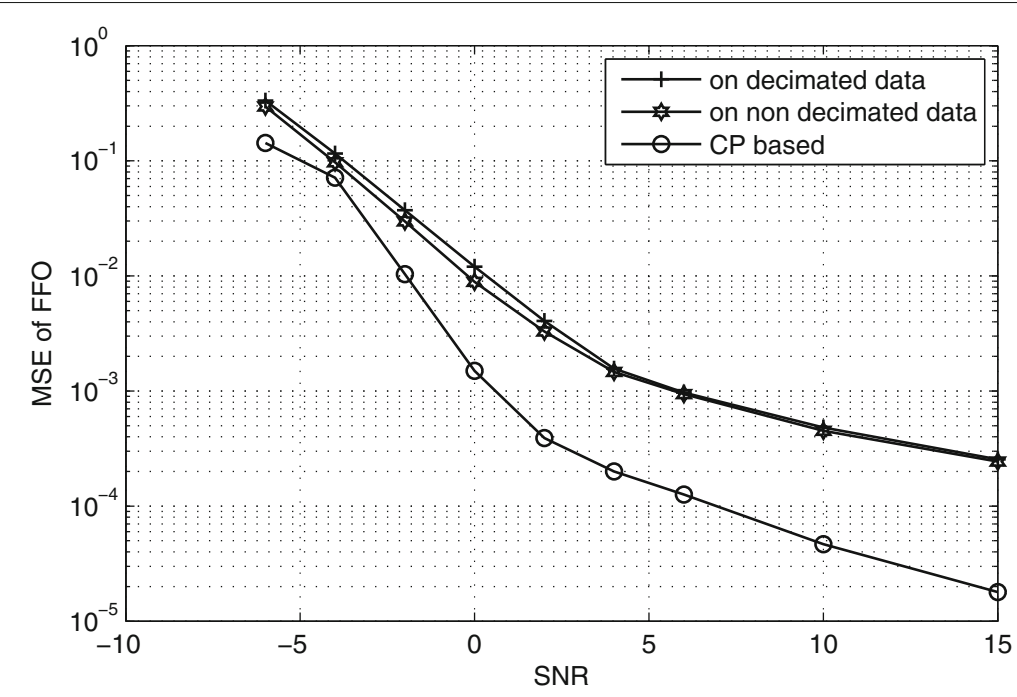

Fig. 10 MSE of the FFO estimators 


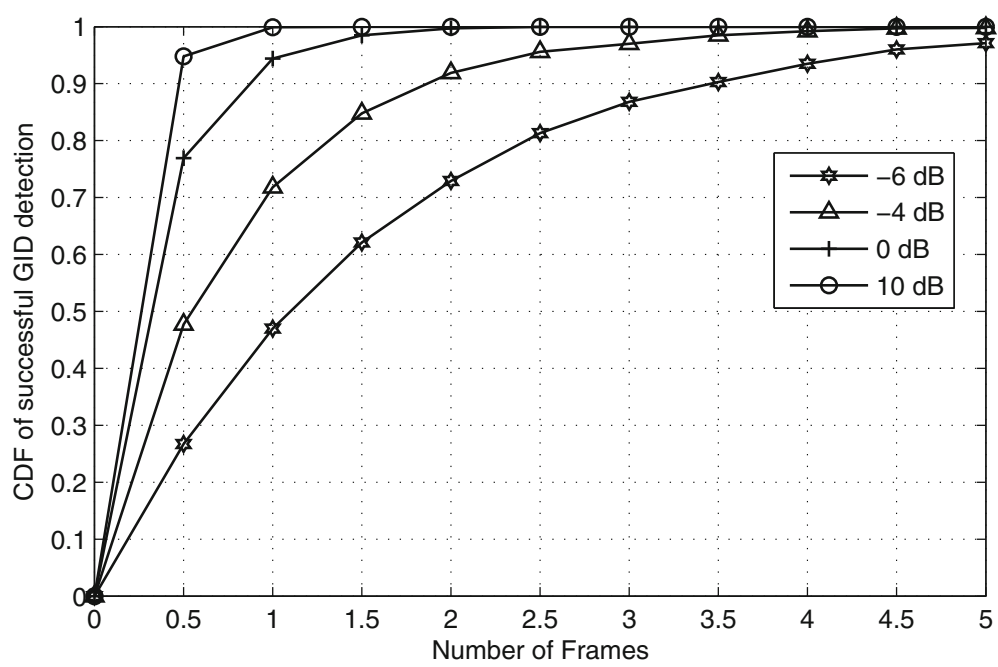

Fig. 11 CDF of the cell-ID detection over number of frames

at 5, module I performance almost approaches to that of module II in the absence of IFO. The CDF of successful detection of SID over the number of frames is presented in Fig. 7. The results show that the chances of detecting SID at $-6 \mathrm{~dB}$ SNR in first half frame is of $70 \%$.

The timing detection probability using module I and module II are presented in Fig. 8. The delay spread of the considered channel is more than half the CP duration. The LPF used for the decimation will also have impact on the timing estimate. So, the detection of timing is considered as successful if the estimate is in the last quarter of the CP. From the results, it is clear that increase in $\lambda$ in turn increases the search window size which makes module I approach towards module II in the absence of IFO.

\subsection{Frequency offset estimation}

The results for the IFO and FFO estimation are presented in this section. The plot shown in Fig. 9 shows the successful detection of IFO over the number of frames. The probability of IFO detection using the first half frame is $92 \%$ at $10 \mathrm{~dB}$ SNR. But at $-6 \mathrm{~dB}$, it takes at least two frames to achieve the same performance. However, there is no need to repeat this estimation every time the UE moves to the vicinity of neighboring BS. The frequency differences among neighboring BSs are negligible compared to the IFO occurring at the local oscillator of the UE, because all neighboring BSs are connected to a master clock by satellite or optical fiber links. The local oscillators operating at the BSs are usually of high accuracy by using controlled crystal oscillators so that the carrier frequencies of the BSs do not deviate.

The results for the mean squared error (MSE) for the FFO estimators are shown in Fig. 10. CP-based estimator exploits the $\mathrm{CP}$ from multiple symbols to eliminate the time-varying channel effect and suppress the noise effect. Unless large portion of $\mathrm{CP}$ is corrupted, the $\mathrm{CP}$ averaging method gives the better performance than the FFO estimator presented in 3.2.1. The results obtained from 3.2.1 are combined with the prior estimates of FFO. The results presented in Fig. 10 are FFO estimates of module II based on single PSS symbol correlation and CP-based estimator. The EVA channel is considered for the simulation whose delay spread is more than half of the $\mathrm{CP}$ length.

\subsection{Cell-ID detection}

Once the timing, CFO, and SID are estimated from the above algorithms, the corresponding parameters have to be compensated. The compensated signal is fed to the SSS

Table 2 Computational complexity

\begin{tabular}{llll}
\hline Algorithm & Multiplications & Additions & Example (multiplications) \\
\hline Module I & $\left(70 N^{D}+60 N^{D}\right) \lambda$ & $\left(70\left(N^{D}-1\right)+60\left(N^{D}-1\right)\right) \lambda$ & 49920 \\
Module II & $N^{D} N_{H F}^{D} \mu$ & $\left(N^{D}-1\right) N_{H F}^{D} \mu$ & 3686400 \\
$C C S A$ & $(70 * 62 \mu+60 * 62 \mu) \lambda$ & $(70 * 61 \mu+60 * 61 \mu) \lambda$ & 72540 \\
DC & $N_{H F}^{D} N^{D}+L_{C P} * 70+(70 * 62 * 3 \mu)$ & $N_{H F}^{D}\left(N^{D}-1\right)+\left(L_{C P}-1\right) * 70+(70 * 61 \mu)$ & 1277940 \\
$M L A$ & $N N_{H F} \mu$ & $(N-1) N_{H F} \mu$ & 943718400 \\
\hline
\end{tabular}


detector which is based on the partial differential correlator [17]. Finally, cell-ID is obtained from the estimates of SID and GID. The CDF of successful detection of cell-ID over number of frames is shown in Fig. 11. The results suggest that for any cell edge user with $-6 \mathrm{~dB}$ SNR, it takes 5 frames on an average to detect cell-ID.

\subsection{Complexity analysis}

The complexity of the receiver in terms of the number of complex multiplications and additions is presented in this section. The above results indicate performance increase with increase in computations. But the joint module I and module II set of algorithms helps in balancing the receiver performance and complexity. Module I is a symbol-bysymbol search method for SID and timing. Module I algorithm works in the absence of large frequency offsets specifically integer offsets. However, if module I fails in detecting the cell-ID, UE try to estimate the cell-ID based on module II. Module II is based on sample-bysample search and as well includes the IFO hypotheses. Both the algorithms are employed with the decimation section to reduce the number of computations. The number of computations of the two modules is presented in Table 2 per each SID choice. $\mu$ is the set of hypotheses considered for IFO detection. $N_{H F}^{D}$ and $N_{H F}$ is the decimated and non-decimated number of samples of half a frame. $N^{D}$ is the decimated OFDM symbol duration excluding $\mathrm{CP}$.

The computation complexities of different algorithms are also furnished in Table 2. The numericals presented in the table do not include the computations needed for FFT. $\lambda, V$, and $\mu$ are considered as 1,16 , and 3 , respectively, for the examples provided in the table. But in CCSA and DC, the PSS search is done in frequency domain posing additional complexity due to the FFT operation. Module I is the least complex of all the algorithms presented which can be observed from the examples from the table.

\section{Conclusions}

A robust cell search procedure that consists of joint operation of two modules is proposed and studied based on the simulations. Both the modules estimate the timing and SID using cross-correlation approach. Module I symbolby-symbol search technique which does not include the estimation of IFO is computationally less complex compared to that of module II. In module II, received signal is diagnosed over every sample to estimate timing and IFO jointly. IFO values are hypothesized over finite possibilities and detected based on the correlation with the PSS sequences. The cost of computations in module II is justified by its performance over module I. Efforts have been made to reduce the computation burden by operating over the decimated samples. The presented cell search operation gives the complete solution with perfect balance of performance and the computational complexity. Simulations have been carried out over the frequency-selective channels of different delay spreads confining to the LTE environment with $20 \mathrm{MHz}$ BW.

\section{Acknowledgements}

The authors would like to thank CCRAN project, Miety, Govt. of India for sponsoring this research work.

\section{Authors' contributions}

$M R$ is the main author of the current paper. MR and KK contributed to the conception and design of the study. SD contributed to the structuring and reviewing of the manuscript. All authors read and approved the final manuscript.

\section{Competing interests}

The authors declare that they have no competing interests.

\section{Publisher's Note}

Springer Nature remains neutral with regard to jurisdictional claims in published maps and institutional affiliations.

Received: 25 October 2016 Accepted: 14 May 2017

Published online: 31 May 2017

\section{References}

1. 3GPP, TS 36.101 User equipment (UE) radio transmission and reception. Tech Report

2. E Dahlman, S Parkvall, J Skold, P Beming, 3G Evolution HSPA and LTE for Mobile Broadband. (Academic Press, MA, 2008)

3. 3GPP, TS 36.211 Phys- Ical Channels and Modulation. Tech Report

4. D Bai, C Park, J Lee, H Nguyen, J Singh, A Gupta, Z Pi, Z kim, C Lim, LTE-advanced modem design: challenges and perspectives. IEEE Commun. Mag. 50, 178-186 (2012)

5. T Pollet, M VanBladel, M Moenclaey, BER sensitivity of OFDM systems to carrier frequency offset and Wiener phase noise. IEEE Trans. Commun. 43, 191-193 (1995)

6. M Schmidl, DC Cox, Robust frequency and timing synchronization for OFDM. IEEE Trans. Commun. 45, 1613-1621 (1997)

7. JJ VandeBeek, M Sandell, PO Borjesson, ML estimation of time and frequency offset in OFDM system. IEEE Trans. Signal Process. 45 1800-1805 (1997)

8. CMa, H Cao, P Lin, A low-power low-cost design of primary synchronization signal detection. IEEE Trans. VLSI Syst. 20, 1161-1166 (2011)

9. K Manolakis, DMG Estevez, V Jungnickel, W Xu, C Drewes, in Proceedings of Wireless Comm and Net Conf (WCNC). A closed concept for synchronization and cell search in 3GPP LTE systems (IEEE, Budapest, 2009), pp. 1-6

10. M Morelli, M Moretti, A robust scheme for pss detection and integer frequency offset recovery in LTE systems. IEEE Trans. Wireless Commun. 15, 1353-1363 (2016)

11. JC Lin, YT Sun, HV Poor, Initial synchronization exploiting inherent diversity for the LTE sector search process. IEEE Trans. Wireless Commun. 15, 1114-1128 (2016)

12. SL Su, YC Lin, YJ Fan, Joint sector identity and integer part of carrier frequency offset detection by phase-difference in long term evolution cell search process. IET Commun. 7, 950-959 (2013)

13. J Wang, L Zhang, Method, receiver and system for signal synchronization, (2016). US 9337994

14. ITU-R, guidelines for evaluation of radio transmission technologies for IMT-2000. Tech Report

15. Ericsson, Nokia, Motorola, Rohde, Schwarz, R4-070572: proposal for LTE channel models. Tech Report

16. WC Jakes, Microwave mobile communications. (Wiley, New York, 1974)

17. JI Kim, JS Han, HJ Roh, HJ Choi, in Conf on Comm and Information Technology. SSS detection method for initial cell search in 3GPP LTE FDD/TDD dual mode receiver (IEEE, Korea, 2009), pp. 199-203 